\title{
Postulation of Canonical Curves in $\mathbb{P}^{3}$
}

\author{
Mei-Chu Chang* \\ Department of Mathematics, University of Michigan, and University of South Carolina, \\ Columbia, SC 29208, USA
}

A non-singular curve $Y \subset \mathbb{P}^{3}$ of genus $g$ is said to be canonical if its plane section is a canonical divisor, i.e. if the canonical bundle $K_{Y} \simeq O_{Y}(1)$. In other words, $Y \subset \mathbb{P}^{3}$ is the projection of its canonical image in $\mathbb{P}^{g-1}$. Thus the canonical curves of given genus form a single irreducible family. Now recall that a curve $Y \subset \mathbb{P}^{3}$ is said to have maximal rank if the restriction maps: $H^{0}\left(O_{\mathbb{P}^{3}}(n)\right) \rightarrow H^{0}\left(O_{Y}(n)\right)$ have maximal rank for all $n$. Given any family of curves in $\mathbb{P}^{3}$, it is a natural question whether its general member has maximal rank. While one usually expects the answer to be "yes", the case of canonical curves is somewhat intriguing in that in the first two nontrivial cases, namely those of genus 5 and 6, the answer turns out to be "no" (proofs were given by Gruson and Peskine [2] and Hartshorne and Sols [3]). Recently the question was taken up by Ballico [1], who showed that a general canonical curve of genus $g=7,8,9$ or 11 has maximal rank.

In this note we prove the following

Theorem. A general canonical curve of genus $g \geqq 7$ in $\mathbb{P}^{3}$ has maximal rank.

The idea of the proof is rather simple. We degenerate a canonical curve to the union of a configuration of conics plus a canonical curve $Y_{0}$ of smaller genus, then crush the conics to a plane curve with embedded points. By suitably manipulating the configuration, we can arrange that any surface containing the union must contain the plane as a component. So after peeling off the plane, we only need to show that $Y_{0}$ and the points $\Delta$ arising from the embedded points are not contained in any surface of smaller degree; in other words, we need to show that $\Delta$ and some hyperplane section of $Y_{0}$ impose independent conditions on certain linear systems.

Lemma 1. Given a general canonical curve $Y_{0}$ and a general 2-secant conic $C$, there is a family of curves whose general member is canonical, and whose special member is $Y_{0} \cup C$.

Proof. It suffices to prove the existence of such $Y_{0}$ and $C$. Consider a oneparameter family $\mathscr{Y} / B$ of abstract curves such that a general fibre of $\mathscr{Y}$ has genus $g$ and a special one is the union of a curve of genus $g-1$ and a rational curve $R$

* Partially supported by a Rackham fellowship at the University of Michigan 
intersecting it in 2 points. Embed each fibre $Y$ by the linear system $\left.K_{\mathscr{y} / B}(-R)\right|_{Y}$, then project to $\mathbb{P}^{3}$.

Let "Configuration (*)" be the following

$$
Y=Y_{0} \cup C_{1} \cup \ldots \cup C_{\delta},
$$

where $Y_{0}$ is a general canonical curve of genus $g_{0}=g-\delta$ and $C_{i}$ 's are conics such that $\#\left(C_{1} \cap Y_{0}\right)=\#\left(C_{i} \cap\left(Y_{0} \bigcup_{j=1}^{i-1} C_{j}\right)\right)=2$ for $i \geqq 2$.

Corollary 1.1. It suffices to prove the theorem for $Y$ as in Configuration (*).

Lemma 2. A general canonical curve of genus $g \geqq 7$ has a general set of points as a hyperplane section.

Proof. Given a set of $2 g-2$ general points on a plane, we can take $g_{0}=7$ in Configuration (*) such that $Y_{0}$ has 12 of the points as a hyperplane section (This follows from the construction in Proposition 6) and each $C_{i}$ passes through 2 points.

The next lemma is a version of the classical fact "nodes impose independent conditions" for reducible curves.

Lemma 3. The intersection points $\Delta$ of $\delta$ plane curves $\bar{C}=\bigcup_{i=1}^{\delta} C_{i}$, where $C_{i} \subset H$ has degree $d_{i}$, impose independent conditions on plane curves of degree $k \geqq d-2:=\sum_{i=1}^{\delta} d_{i}-2$.

Proof. Let $\pi$ be the natural map: $\coprod_{i=1}^{\delta} C \rightarrow \bar{C}$, and let $\Delta_{i}=\pi^{-1}(\Delta) \cap C_{i}$. Then we have $\left|O_{\bar{C}}(k)-\Delta\right|=\left|\oplus O_{C_{i}}(k)-\Delta_{i}\right|$.

To compute the dimension of the right hand side, we notice that

$$
\oplus O_{C}\left(d_{i}-3\right)\left(\Delta_{i}\right)=\oplus K_{C_{i}}\left(\Delta_{i}\right)=\pi^{*} K_{\bar{C}}
$$

and

$$
\pi^{*} K_{\bar{C}}=\pi^{*} O_{\bar{c}}(d-3)=\oplus O_{C_{i}}(d-3) \text {. }
$$

Hence $\left|\oplus O_{C_{i}}(k)-\Delta_{i}\right|=\left|\oplus O_{C_{i}}\left(k-d+d_{i}\right)\right|$.

Now straightforward computation gives $\operatorname{dim}\left|O_{\bar{C}}(k)-\Delta\right|=h^{0}\left(O_{\bar{C}}(k)-\# \Delta\right.$.

Before starting the proof, we introduce the following notations. For each $g$, let $Y$ be a canonical curve of genus $g$. We define

$$
\begin{gathered}
n_{g}:=\min \left\{n \mid \chi\left(I_{Y}(n)\right) \geqq 0\right\} \\
g_{n}:=\max \left\{g \mid n_{g}=n\right\} \\
\gamma_{g}:=\chi\left(I_{Y}\left(n_{g}\right)\right) .
\end{gathered}
$$

Observing that $\left(\begin{array}{c}n+3 \\ 3\end{array}\right)=n(2 g-2)+1-g+\gamma_{g}=(2 n-1)(g-1)+\gamma_{g}$, we have, e.g., $g_{n} \sim\left[\frac{n^{2}}{12}+\frac{13 n}{24}+\frac{57}{48}\right]+1, \gamma_{g_{n}-\delta}=\gamma_{g_{n}}+\delta(2 n-1)$, and $\gamma_{g_{n}}<2 n-1$. Through the 
rest of the paper, we fix $g$ and let $n:=n_{g}$ and $\gamma:=\gamma_{g}$. We divide the proof of the theorem into two parts.

Part I. We claim that $h^{0}\left(I_{Y}(n)\right)=\gamma$ for some $Y$ as in Configuration (*). It is sufficient to show that $H^{0}\left(I_{Y \cup R}(n)\right)=0$ where $R$ is a set of $\gamma$ points to be chosen.

Let $X:=Y_{0} \cap\left(\bigcup_{i=1}^{\delta} C_{i}\right)$ and $x:=\# X$. Note that $2 \delta \geqq x \geqq 2$. We specialize $Y$

further so that $Y=\bar{\Sigma} \cup Y_{0}$ where $\bar{\Sigma}$ is a plane curve $\Sigma C H$ of degree $d=2 \delta$ (respectively $d=2 \delta+1$, if $g=g_{n}$ ) plus embedded points at some of the nodes of $\Sigma$, $Y_{0}$ has the property that $H^{1}\left(I_{Y_{0}}(n-1)=0\right.$, and $\#\left(\Sigma \cap Y_{0}\right)=x, 2 \leqq x \leqq 2 \delta$ (resp. $1 \leqq x \leqq 4 \delta$ ). We can do so because if $g \neq g_{n}, \bar{\Sigma}$ is just the limit of $\bigcup_{i=1}^{\delta} C_{i}$; if $g=g_{n}$, we first specialize $Y_{0}$ as in Configuration (*) so that $Y_{0}=\bar{\Sigma}_{1} \cup L \cup Y^{\prime}$ where $\bar{\Sigma}_{1} \cup L$ is a union of $\delta_{1}$ conics, $1 \leqq \delta_{1} \leqq \delta$, and $L$ intersects $Y_{1}=\bar{\Sigma}_{1} \cup Y^{\prime}$ in $x_{1}$ points $1 \leqq x_{1} \leqq 2 \delta_{1}$, with normal crossings. Now specialize $\cup C_{i}$ in a plane and take $\bar{\Sigma}=Y \backslash Y_{1}$. The new $Y_{0}$ is $Y_{1}$ which is a "canonical" (reducible) curve minus a line.

If $F_{n} \in H^{0}\left(I_{Y \cup R}(n)\right)$ and $F_{n} D H$, then $F_{n} \cap H$ is a plane curve containing $\Sigma$ and $\left(R \cup Y_{0}\right) \cap H \backslash X$, i.e. $\left(R \cup Y_{0}\right) \cap H \backslash X$ is contained in a plane curve of degree $n-d$. By Lemma 4 below, we can choose $x$ and $\delta$, such that $s=h^{0}\left(O_{\mathbb{P}_{2}}(n-d)\right)-\left(2 g_{0}-2-x\right)$ is between 0 and $\gamma$. So let a subset $S$ of $s$ points of $R$ be general on $H$, then $S \cup\left(Y_{0} \cap H\right) \backslash X$ can not be contained in any plane curve of degree $n-d$. Therefore $F_{n} \supset H$ and the problem reduces to proving $Y_{0} \cup(R \backslash S) \cup \Delta$ is not contained in any surface of degree $n-1$, where $\Delta$ is a subset of intersecting points of the plane curves. Part I is proved, if the following claim is true.

Claim. $\Delta$ impose independent conditions on $H^{0}\left(I_{Y_{0}}(n-1)\right)$.

\section{Proof of the Claim}

Case 1. $Y_{0}$ is canonical. By induction $Y_{0}=Y_{1} \cup \widetilde{\Sigma_{0}}$ where $Y_{1}$ is either a canonical curve $Y^{1}$ or a canonical curve $Y^{1}$ minus a line $L_{0}$ and $\tilde{\Sigma}_{0}$ is a plane curve $\Sigma_{0} C H$ of degree $d_{0}$ with embedded points. Induction hypothesis implies that $\Delta_{0}$ impose independent conditions on $H^{0}\left(I_{Y_{1}}\left(n_{g_{0}}-1\right)\right)$, i.e., $H^{1}\left(I_{Y_{1} \cup A_{0}}\left(n_{g_{0}}-1\right)\right)=0$. Since $n_{g_{0}}-1 \leqq n-2$, we have $H^{1}\left(I_{Y_{1} \cup A_{0}}(n-2)\right)=0$. The long cohomology sequence of the exact sequence.

$$
0 \rightarrow I_{Y_{1} \cup A_{0}}(n-2) \rightarrow I_{Y_{0}}(n-1) \rightarrow I_{Y_{0} \cap H}(n-1)=I_{Y_{0} \cap H / \Sigma_{0}}\left(n-1-d_{0}\right) \rightarrow 0
$$

gives the surjection $H^{0}\left(I_{Y_{0}}(n-1)\right) \rightarrow H^{0}\left(I_{Y_{0} \cap H / \Sigma_{0}}\left(n-1-d_{0}\right)\right)$. The claim now follows from Lemmas 2 and 3.

Case 2. $Y_{0}=\bar{\Sigma}_{1} \cup Y^{\prime}$ (as in Part 1 ) is a "canonical" curve minus a line $L$. Then the same process as in Case 1 gives that $\bar{\Sigma}_{0}$ contains the limit of $L \cup \widetilde{\Sigma}_{1}$ and $\Delta$ imposes independent conditions on $H^{0}\left(I_{Y_{0} \cup L}(n-1)\right)$, hence on the larger space $H^{0}\left(I_{Y_{0}}(n-1)\right)$.

Part II. We claim that $H^{0}\left(I_{Y}(\leqq n-1)\right)=0$ for some canonical curve $Y$ of genus $g$. Let $Y=Y^{\prime} \cup C$, where $Y^{\prime}$ has genus $g-1$. If $n_{g-1}=n$, then the claim follows from the induction hypothesis on $Y$. Otherwise $n_{g-1}=n-1$ and Corollary 5 implies that 
in Configuration $(*)$ we can choose $x$ and $\delta$ such that both ends of the following sequence are 0 :

$$
0=H^{0}\left(I_{Y_{0} \cup A}(n-2)\right) \rightarrow H^{0}\left(I_{Y}(n-1)\right) \rightarrow H^{0}\left(I_{Y_{0} \cap H \backslash \Sigma}(n-1-d)\right)=0 .
$$

Finally the theorem follows from Parts I, II and Castelnuovo lemma.

Lemma 4. There exist $x, x^{\prime}$ and $\delta, 2 \leqq x \leqq 2 \delta$, and $1 \leqq x^{\prime} \leqq 4 \delta$ such that $s(x)=h^{0}\left(O_{\mathbb{P}_{2}}(n-2 \delta)\right)-(2 g-2 \delta-2-x)$ is between 0 and $\gamma=h^{0}\left(I_{Y}(n)\right)$ if $g \neq g_{n}$. And either $s(x)$ or $s^{\prime}\left(x^{\prime}\right)=h^{0}\left(O_{\mathbb{P}_{2}}(n-2 \delta-1)\right)-\left(2 g-2 \delta-2-x^{\prime}\right)$ is 0 if $g=g_{n}$.

Proof. If $g \neq g_{n}$, let $b=\frac{g_{n}-g-1}{n}$ and $\delta=: c n$. We have $\gamma=\gamma_{g_{n}}+(2 n-1)$ $+b n(2 n-1)$ and $g-1=g_{n}-b n-2 \leqq \frac{n^{2}}{12}+\frac{13 n}{24}+\frac{9}{48}-b n$. Comparing the coefficients of $n^{2}$ and $n$, we have $s(x) \geqq 0$ if $c \leqq \frac{1}{2}-\frac{1}{\sqrt{12}}$. Similarly we have $\gamma \geqq s(x)$ if $c \geqq \frac{1}{2}$ $-\frac{1}{2} \sqrt{4 b+\frac{1}{3}}$. Hence, we can take $\delta=\left[\left(\frac{1}{2}-\frac{1}{\sqrt{12}}\right) n\right]$ and $x=\delta$.

Now, if $g=g_{n}, \delta=\left[\left(\frac{1}{2}-\frac{1}{\sqrt{12}}\right) n\right]$ always gives $s(0) \geqq 0$. If $s(2) \geqq 1$ and $s^{\prime}(0)$ $\leqq-4 \delta$, then $s(2)-s^{\prime}(0)=n-2 \delta+1 \geqq 4 \delta+1$, which contradicts to the choice of $\delta$. Therefore either $s(2)=0$ or $s^{\prime}\left(x^{\prime}\right)=0$.

Corollary 5. If $g=g_{n-1}+1$, then there exist $x$ and $\delta$ such that $h^{0}\left(O_{\mathbb{P}_{2}}(n-1-2 \delta)\right)$ $-(2 g-2 \delta-2-x)=0$.

Proposition 6. There is a canonical curve of genus 7 having maximal rank.

Proof. Let $Y_{4}$ be an abstract curve of genus $4, Q$ be a general member of a $g_{3}^{1}$ of $Y_{4}$, and $2 P_{2}+P_{3}$ a nonreduced member of the $g_{3}^{1}$. In the 1-parameter family of abstract curves $\mathscr{Y} / B$ let the special fibre $Y=Y_{4} \cup\left(\bigcup_{1}^{3} C_{i}\right)$ where $C_{1}$ is elliptic intersecting $Y_{4}$ at $Q, C_{i}$ is rational intersecting $Y_{4}$ at $P_{i}$ for $i=2$ and 3. A general projection of the linear system $\left|K_{\text {oy/B }}\left(-3 C_{2}-2 C_{3}\right)\right|$ gives the desired embedding, namely $Y=Y_{4} \cup\left(\bigcup_{1}^{3} C_{i}\right)$ where $C_{1} \subset H_{1}$ is a plane cubic, $C_{2} \subset H_{2}$ is a conic, $C_{3}$ is a line and $Y_{4}$ is a canonical curve of genus 4 . To prove maximal rank, it suffices to show $H^{0}\left(I_{Y \cup R}(5)\right)=0$ where $R$ is a set of 2 points lying on a plane $H_{3} \supset C_{3}$. We peel off $H_{1}$, then $H_{2}$, then $H_{3}$ and finally use the fact that $h^{0}\left(I_{Y_{4}}(2)\right)=1$.

\section{References}

1. Ballico, E.: On the postulation of canonical curves in $\mathbb{P}^{3}$. Ark. Mat. 22, 139-151 (1984)

2. Gruson, L., Peskine, C.H.: Genre des courbes de l'espace projective. Algebraic geometry. Lect. Notes Math. 678. Berlin, Heidelberg, New York: Springer 1978

3. Hartshorne, R., Sols, I.: Stable rank 2 vector bundles on $\mathbb{P}^{3}$ with $c_{1}=1, c_{2}=2$. J. Reine Angew. Math. 325, 145-152 (1981) 